\title{
Characterization of Some Groups of Gram-Negative Nonfermentative Bacteria by the Carbon Source Alkalinization Technique
}

\author{
R. MARTIN, ${ }^{1} \dagger^{*}$ P. S. RILEY, ${ }^{2}$ D. G. HOLLIS, ${ }^{3}$ R. E. WEAVER, ${ }^{3}$ AND M. I. KRICHEVSKY ${ }^{4}$ \\ School of Public Health, University of North Carolina, Chapel Hill, North Carolina 27514 ${ }^{1} ; 681$ S. King \\ Street, Honolulu, Hawaii $98613^{2}$; Centers for Disease Control, Atlanta, Georgia 30333; and Microbial \\ Systematics Unit, National Institutes of Dental Research, Bethesda, Maryland 20014
}

Received 8 December 1980/Accepted 16 March 1981

\begin{abstract}
A total of 541 gram-negative nonfermentative bacterial strains comprising 26 species and unclassified groups were characterized by routine diagnostic and carbon substrate alkalinization techniques. These microorganisms were tested for the ability to cause alkalinization of a basal medium on a total of 217 substrates. We found that 58 carbon substrates had some discriminatory potential. We also performed 30 routine diagnostic tests. The results of these studies were evaluated by numerical taxonomy techniques. A cluster analysis of the results by the Jaccard coefficient method identified 30 clusters at the $45 \%$ level. We identified 39 tests that separated most of the groups. Groups of similar organisms or organisms that were difficult to identify were analyzed, and tests that were differential were identified. Because of variability within the clusters, further studies utilizing deoxyribonucleic acid-deoxyribonucleic acid homologies should be undertaken.
\end{abstract}

The nonfermentative gram-negative bacteria are distributed widely in nature and have been isolated from soil, water, and medical devices, as well as from clinical specimens $(1,3,10,12,28)$. In recent years, the number of diagnoses of infections caused by nonfermentative bacteria has increased, largely because of a heightened awareness of the possible roles of these bacteria as pathogens rather than as contaminants $(2,4$, $6,7-9)$. The widespread use of antibiotics and other chemotherapeutic agents in the treatment of diseases has played a major role in the increased frequency of infection by these organisms because of the disruption of the normal flora (4). Another factor is the increase in the number of debilitated people with chronic diseases that may impair the immunological defense mechanisms. Because of the increased incidence of infections due to the nonfermentative bacteria, correct identification of these organisms is important.

The increasing importance of the nonfermentative bacteria has prompted a search for additional rapid, reliable, specific distinguishing tests to supplement or replace the currently used routine diagnostic tests. Carbon source utiliza-

† Present address: Bureau of Disease Control and Laboratory Services, Houghton, MI 49931.

TABLE 1. Nonfermentative gram-negative bacteria studied

\begin{tabular}{lc}
\hline \multicolumn{1}{c}{ Taxon } & $\begin{array}{c}\text { No. of } \\
\text { strains }\end{array}$ \\
\hline Pseudomonas denitrificans & 22 \\
Pseudomonas testosteroni & 23 \\
Pseudomonas acidovorans & 25 \\
Pseudomonas alcaligenes & 17 \\
Pseudomonas pseudoalcaligenes & 17 \\
Pseudomonas diminuta & 27 \\
Pseudomonas putrefaciens biotype 1 & 10 \\
Pseudomonas putrefaciens biotype 2 & 13 \\
Pseudomonas vesicularis & 12 \\
CDC group WO-1 & 12 \\
Alcaligenes faecalis I & 23 \\
Alcaligenes faecalis II & 23 \\
Alcaligenes odorans & 23 \\
Alcaligenes denitrificans & 23 \\
CDC group IVc-2 & 25 \\
CDC group IVe & 24 \\
Bordetella bronchiseptica & 24 \\
Flavobacterium odoratum & 23 \\
CDC group M-5 & 22 \\
CDC group M-6 & 24 \\
Acinetobacter calcoaceticus subsp lwoffi & 21 \\
Moraxella urethralis & 25 \\
Moraxella osloensis & 22 \\
Moraxella nonliquefaciens & 23 \\
Moraxella phenylpyruvica & 19 \\
Moraxella atlantae & 19 \\
\hline
\end{tabular}


tion, which is an alternative method for identifying and taxonomically classifying bacteria, has been used by a number of investigators $(12,15-$ $19,22-24)$.

This report describes the characterization of 26 species and unclassified groups of nonfermentative gram-negative bacteria (primarily nonsaccharolytic organisms) maintained in the culture collection of the Special Bacteriology Section, Centers for Disease Control. Because of the large amount of data generated by the carbon substrate tests and the diagnostic tests, it was necessary to use a computer programmed for sorting data by numerical taxonomy techniques.

(The results were taken from a thesis pre- sented by R.M. to the University of North Carolina.)

\section{MATERIALS AND METHODS}

Bacterial cultures. The organisms studied were obtained from the stock culture collection maintained by the Special Bacteriology Section, Centers for Disease Control. They were identified from isolates submitted to this laboratory for further investigation (Table 1). The designations of the unclassified groups are arbitrary designations which are used in the Special Bacteriology Section.

Diagnostic studies. The biochemical tests used were those routinely used in the Special Bacteriology Section for the characterization of microorganisms submitted for identification. The methods for preparing the media and performing the tests have been

CLUSTERS

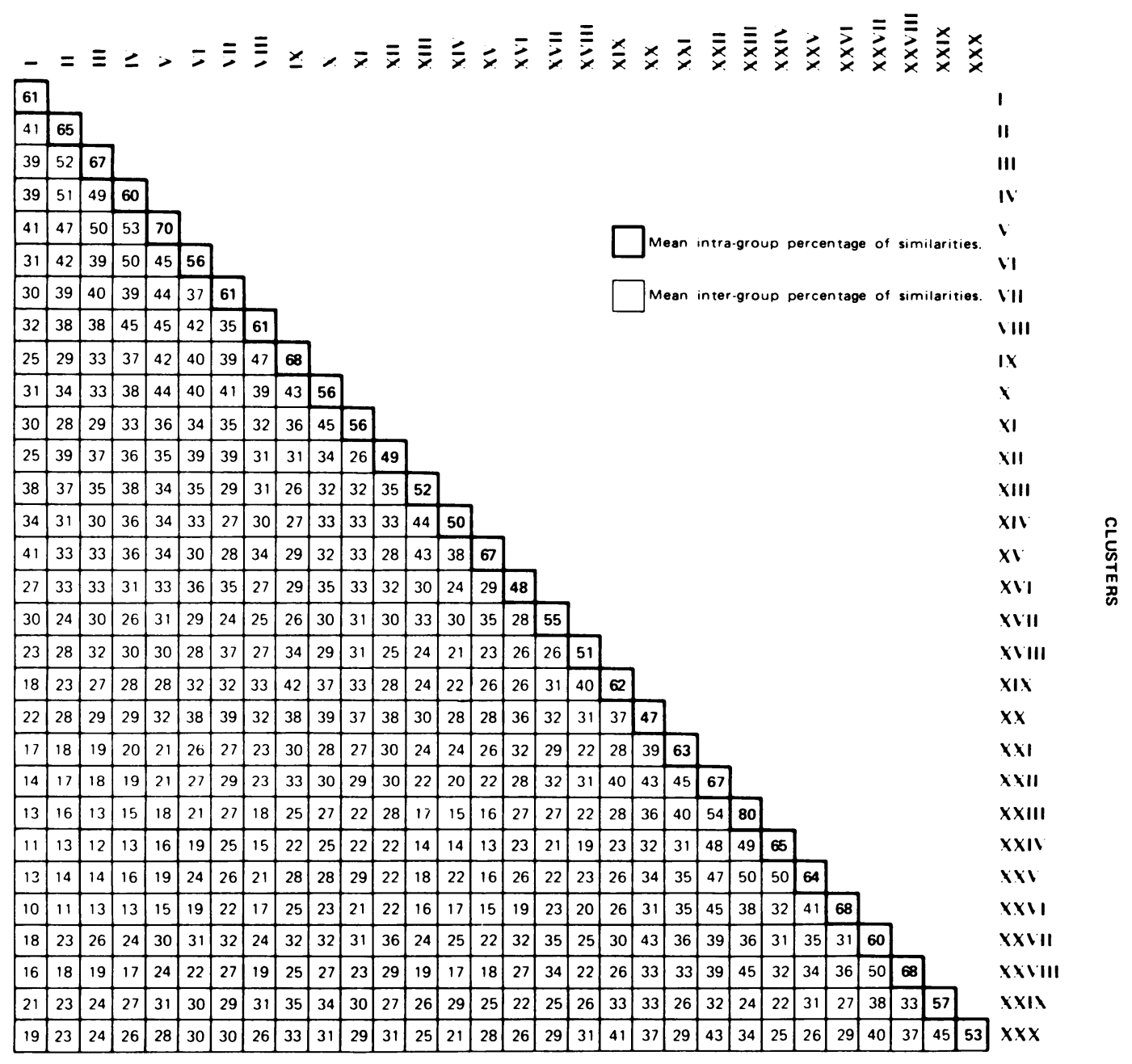

Fig. 1. Mean inter-and intragroup similarities of 30 clusters delineated at the $45 \%$ level of similarity by the Jaccard coefficient. 
TABLE 2. Contents of the 30 clusters determined by numerical taxonomic methods.

\begin{tabular}{|c|c|c|c|c|c|}
\hline Cluster & Organism & $\begin{array}{l}\text { No. of } \\
\text { strains }\end{array}$ & Cluster & Organism & $\begin{array}{l}\text { No. of } \\
\text { strains }\end{array}$ \\
\hline I & P. putrefaciens biotype 1 & 6 & $\mathrm{XX}$ & P. denitrificans & 6 \\
\hline & P. putrefaciens biotype 2 & 12 & & p. diminuta & 1 \\
\hline \multirow[t]{4}{*}{ II } & P. acidovorans & 22 & & P. alcaligenes & 1 \\
\hline & P. testosteroni & 9 & & P. pseudoalcaligenes & 1 \\
\hline & A. faecalis II & 1 & & A. denitrificans & 1 \\
\hline & P. alcaligenes & 1 & XXI & P. vesicularis & 10 \\
\hline \multirow[t]{3}{*}{ III } & CDC group IVc-2 & 21 & XXII & M. osloensis & 15 \\
\hline & A. faecalis I & 7 & & CDC group M-5 & 20 \\
\hline & A. denitrificans & 2 & & M. atlantae & 3 \\
\hline \multirow[t]{2}{*}{ IV } & A. faecalis I & 13 & & A. calcoaceticus subsp. lwoffi & 3 \\
\hline & A. denitrificans & 10 & & P. alcaligenes & 3 \\
\hline \multirow[t]{3}{*}{ V } & B. bronchiseptica & 25 & & P. denitrificans & 1 \\
\hline & CDC group IVc-2 & 1 & & M. urethralis & 1 \\
\hline & A. faecalis I & 3 & & M. nonliquefaciens & 1 \\
\hline \multirow[t]{2}{*}{ VI } & A. denitrificans & 9 & XXIII & M. nonliquefaciens & 20 \\
\hline & A. faecalis I & 4 & & M. osloensis & 1 \\
\hline VII & P. testosteroni & 11 & & CDC group M-5 & 1 \\
\hline \multirow[t]{2}{*}{ VIII } & A. odorans & 21 & XXIV & CDC group M-6 & 20 \\
\hline & P. alcaligenes & 1 & & M. nonliquefaciens & 1 \\
\hline \multirow[t]{2}{*}{ IX } & A. faecalis II & 14 & XXV & M. osloensis & 2 \\
\hline & P. alcaligenes & 1 & & M. nonliquefaciens & 2 \\
\hline \multirow[t]{5}{*}{$\mathbf{X}$} & P. pseudoalcaligenes & 11 & & CDC group M-6 & 2 \\
\hline & $P$. alcaligenes & 4 & & P. diminuta & 1 \\
\hline & A. denitrificans & 1 & & M. urethralis & 1 \\
\hline & A. faecalis I & 1 & & CDC group M-5 & 1 \\
\hline & P. denitrificans & 1 & & CDC group WO-1 & 1 \\
\hline \multirow[t]{2}{*}{ XI } & P. denitrificans & 5 & XXVI & M. atlantae & 14 \\
\hline & P. alcaligenes & 1 & & CDC group M-6 & 1 \\
\hline \multirow[t]{2}{*}{ XII } & P. testosteroni & 2 & XXVII & CDC group IVe & 20 \\
\hline & P. alcaligenes & 4 & & P. denitrificans & 1 \\
\hline XIII & P. diminuta & 14 & & P. pseudoalcaligenes & 1 \\
\hline XIV & P. diminuta & 3 & XXVIII & M. phenylpyruvica & 17 \\
\hline XV & P. diminuta & 4 & & CDC group IVe & 1 \\
\hline XVI & CDC group WO-1 & 9 & XXIX & M. urethralis & 15 \\
\hline XVII & F. odoratum & 21 & & CDC group IVe & 1 \\
\hline XVIII & A. calcoaceticus subsp. lwoffi & 10 & $\mathrm{XXX}$ & M. urethralis & 4 \\
\hline & P. denitrificans & 1 & & CDC group IVe & 1 \\
\hline XIX & A. calcoaceticus subsp. lwoffi & 7 & & A. calcoaceticus subsp. lwoffi & 1 \\
\hline
\end{tabular}

described previously $(5,20,25)$. Most agar slant culture media were inoculated with 1 drop of an 18- to 24-h heart infusion broth culture. Phenylalanine agar was inoculated with a loopful of culture material.

Substrate alkalinization studies. The alkalinization technique was performed by the procedure of Oberhofer and Rowen (15). The substrates were added to the basal medium, Simmons citrate base (Difco Laboratories), to final concentrations of $0.3 \%$. The filter-sterilized substrates were added aseptically to the sterile melted agar base; those substrates not soluble in water were sterilized by autoclaving at $15 \mathrm{lb} /$ in $^{2}$ and $121^{\circ} \mathrm{C}$ for $15 \mathrm{~min}$. The completed media ( $\mathrm{pH}$ 6.8) were dispensed into screw-capped tubes ( 13 by $100 \mathrm{~mm}$ ) and allowed to cool on slant boards. Each agar slant was inoculated with 1 drop of an 18- to 24$h$ heart infusion broth (Difco) culture, and the tubes were incubated at $35^{\circ} \mathrm{C}$. The incubated tubes were examined after 1,2 , and 7 days. A positive result (alkalinization of the medium) was recorded when the color of the medium changed to blue.

The substrates used were acetamide, acetate, cisaconitate, trans-aconitate, adipate, adonitol, B-alanine, $\mathrm{D}$-alanine, L-alanine, alginate, allantoin, $m$-aminobenzoate, 5-aminobutyrate, DL-2-aminobutyrate, $\gamma$ - aminobutyrate, 2 -aminoethanol, $\delta$-aminolevulinate, 4aminosalicylate, $\delta$-amino- $n$-valerate, $\boldsymbol{n}$-amyl alcohol, amylamine, aniline, anthranilate, L-arabinose, arbutin, arginine, ascorbate, asparagine, L-aspartate, azelate, benzoate, benzylamine, benzylformate, betaine, D-biotin, 1,4-butanediol, butanol, butylamine, $n$-butyrate, catechol, cellobiose, cetrimide, cholate, L-(+)-citrulline, creatine, creatinine, L-cysteine, decane, decanoate, dextrin, diacetyl, dihydroxyacetone, 1,3-dihydroxy-2-propanone, dimethylaminobenzaldehyde, docosanoate, dulcitol, eicosane, erucate, erythritol, ethanol, DL-ethionine, ethylene glycol, ethylene glycol monomethyl ether, folate, formamide, formate, fructose, DL-fucose, fumarate, $\gamma$-D-galactonolactone, galactose, galacturonate, gallate, gentisate, gluconate, glucose, glucuronate, glutamate, L-glutamine, gluarate, DL-glycerate, glycerol, glycine, glycogen, glycolate, glyoxylate, $n$-heptane, heptanoate, $n$-hexadecane, $n$-hexane, 1,6-hexanediol, hexanoate, 2-hexanol, hippurate, histamine, $L$-histidine, $m$-hydroxybenzoate, $p$-hydroxybenzoate, DL- $\beta$-hydroxybutyrate, $\beta$-hydroxy- $\beta$-methylglutarate, hydroxy-L-proline, 3-(2-aminoethyl)indolehydrochloride, indole-3-acetate, inositol, isobutanol, isobutyramide, isobutyrate, DL-isoleucine, isopentanol, isophthalate, isopropanol, isovalerate, ita- 
TABLE 3. Characteristics of clusters of selected nonfermentative bacteria

\begin{tabular}{|c|c|c|c|c|c|c|c|c|c|c|c|c|c|c|c|}
\hline \multirow[b]{2}{*}{ Cluster } & \multicolumn{15}{|c|}{$\%$ of positive reactions } \\
\hline & 离 & 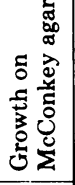 & 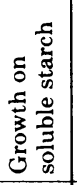 & 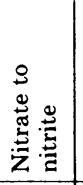 & 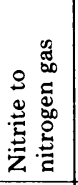 & 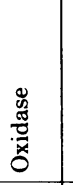 & 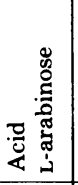 & 莺 & 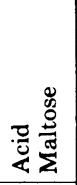 & 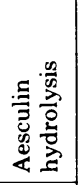 & 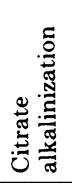 & 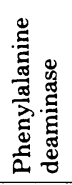 & 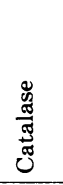 & 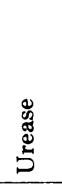 & 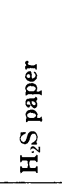 \\
\hline I & 100 & 100 & 83 & 94 & $\mathrm{NT}^{b}$ & 100 & 33 & 17 & 22 & 2 & 11 & NT & 100 & 72 & 100 \\
\hline II & 100 & 100 & 67 & 97 & 0 & 100 & 0 & 0 & 0 & 0 & 100 & NT & 100 & 0 & 39 \\
\hline III & 97 & 100 & 3 & 0 & 0 & 100 & 0 & 0 & 0 & 0 & 100 & NT & 100 & 70 & 33 \\
\hline IV & 96 & 100 & 100 & 59 & 52 & 100 & 0 & 0 & 0 & 0 & 100 & NT & 100 & 0 & 4 \\
\hline V & 100 & 100 & 100 & 80 & 0 & 100 & 0 & 0 & 0 & 0 & 100 & NT & 100 & 84 & 56 \\
\hline VI & 100 & 100 & 100 & 23 & 69 & 100 & 0 & 0 & 0 & 0 & 77 & NT & 100 & 0 & 69 \\
\hline VII & 100 & 100 & 55 & 100 & NT & 100 & 0 & 0 & 0 & 0 & 9 & NT & 100 & 36 & 100 \\
\hline VIII & 86 & 100 & 100 & 9 & 100 & 100 & 0 & 0 & 0 & 0 & 100 & NT & 100 & 0 & 0 \\
\hline IX & 93 & 100 & 100 & 0 & 0 & 100 & 0 & 0 & 0 & 0 & 100 & NT & 100 & 0 & 64 \\
\hline $\mathrm{X}$ & 94 & 100 & 94 & 94 & 50 & 100 & 0 & 0 & 0 & 0 & 56 & NT & 100 & 0 & 94 \\
\hline XI & 100 & 100 & & 100 & 100 & 100 & 50 & 0 & 0 & 0 & 17 & NT & 100 & 0 & 100 \\
\hline XII & 83 & 100 & 67 & 67 & NT & 100 & 0 & 0 & 0 & 0 & 17 & NT & 100 & 17 & 17 \\
\hline XIII & 100 & 100 & 0 & 0 & NT & 100 & 0 & 21 & 0 & 0 & 0 & NT & 100 & 14 & 7 \\
\hline XIV & 100 & 100 & 7 & 0 & NT & 100 & 0 & 0 & 0 & 0 & 0 & NT & 100 & 0 & 33 \\
\hline XV & 100 & 100 & 0 & 0 & NT & 100 & 100 & 50 & 0 & 0 & 0 & NT & 100 & 50 & 0 \\
\hline XVI & 100 & 60 & 0 & 100 & NT & 100 & 0 & 100 & 0 & 0 & 20 & NT & 100 & 60 & 90 \\
\hline XVII & 0 & 100 & 19 & 0 & 100 & 100 & 0 & 0 & 0 & 0 & 0 & NT & 100 & 100 & 38 \\
\hline XVIII & 0 & 91 & 9 & 0 & 100 & 9 & 0 & 0 & 0 & 0 & 46 & NT & 100 & 10 & 64 \\
\hline XIX & 0 & 100 & 43 & 0 & NT & 0 & 0 & 0 & 0 & 0 & 86 & NT & 100 & 0 & 43 \\
\hline XX & 100 & 100 & 10 & 25 & 100 & 100 & 0 & 0 & 0 & 0 & 50 & NT & 100 & 10 & 80 \\
\hline XXI & 90 & 70 & 0 & 0 & NT & 100 & 10 & 100 & 100 & 90 & 0 & NT & 100 & 0 & 40 \\
\hline XXII & 6 & 51 & 0 & 2 & 5 & 94 & 0 & 0 & 0 & 0 & 0 & 61 & 98 & 0 & 70 \\
\hline XXIII & 0 & 0 & 0 & 95 & 0 & 100 & 0 & 0 & 0 & 0 & 0 & 0 & 100 & 0 & 50 \\
\hline XXIV & 0 & 9 & 0 & 100 & NT & 100 & 0 & 10 & 0 & 0 & 0 & NT & 0 & 0 & 86 \\
\hline XXV & 10 & 10 & 0 & 50 & 0 & 100 & 0 & 10 & 0 & 0 & 0 & 50 & 80 & 0 & 90 \\
\hline XXVI & 0 & 100 & 0 & 7 & 0 & 100 & 0 & 0 & 0 & 0 & 0 & NT & 87 & 0 & 75 \\
\hline XXVII & 64 & 91 & 0 & 75 & 100 & 100 & 0 & 0 & 0 & 0 & 18 & NT & 100 & 100 & 32 \\
\hline XXVIII & 6 & 94 & 0 & 94 & NT & 100 & 0 & 0 & 0 & 0 & 0 & 100 & 94 & 100 & 29 \\
\hline XXIX & 0 & 100 & 6 & 0 & 100 & 100 & 0 & 0 & 0 & 0 & 75 & 100 & 100 & 6 & 6 \\
\hline $\mathrm{XXX}$ & 0 & 100 & 0 & 17 & NT & 83 & 0 & 0 & 0 & 0 & 33 & 100 & 100 & 17 & 0 \\
\hline
\end{tabular}

conate, 2 -ketogluconate, $\alpha$-ketoglutarate, kynurenate lactate, laurate, $\mathrm{L}$-leucine, levulinate, linoleate, linolenate, L-lysine monohydrochloride, D-lyxose, maleate, L-malate, D-malate, malonamide, maltose, D-mandelate, L-mandelate, mannitol, mannose, melezitose, melibiose, methanol, L-methionine, methylamine, 4methylvalerate, mucate, myristate, nicotinamide, nicotinate, $n$-nonane, nonanoate, DL-norleucine, DL-norvaline, octadecane, $n$-octane, octanoate, DL-ornithine, orotate, oxalate, palmitate, pantothenate, pentadecanoate, 1-pentadecanol, phenol, phenylacetate, L-phenylalanine, $p$-phenylphenol, phthalate, pimelate, potassium hydrogen phthalate, L-proline, 1-propanol, propionamide, propionate, propylene glycol, protocatechuate, putrescine, pyruvate, D-quinate, 8-quinolinol, raffinose, rhamnose, ribose, D-saccharate, salicin, salicylate, sebacate, sorbate, sorbitol, L-sorbose, spermine, starch, stearate, suberate, succinate, succinamide, sucrose, sulfanilate, tannate, $d$-tartarate, $m$-tartarate, terephthalate, testosterone, thiamine, L-threonine, trehalose, tributyrin, DL-tryptophane, Tween 20 , Tween 40, Tween 60, Tween 80, Tween 85, undecane, undecanoate, urate, valeramide, valerate, DL-valine, vanillate, xanthine, xylan, xylose.

Data processing and analysis. Data were coded in binary form according to the format of Rogosa et al. (21), punched on cards, verified, and proofread. Errors were also checked by a computer, using the CREATE program (13). The QUERY computer program (13) was used to arrange the data suitably for input into the numerical taxonomy program TAXON (a modified version of TAXANS; kindly supplied by P. H. A. Sneath). The MTRXED program (27) was used to edit data so as to remove strains with more than $35 \%$ missing results, as well as nondifferentiating features with more than $99 \%$ positive, $99 \%$ negative, or $90 \%$ missing results. Degrees of similarity were estimated with the Jaccard coefficient, and cluster analyses were performed by unweighted average linkage sorting. Clusters of strains which were more than 
TABLE 3-Continued

$\%$ of positive reactions ${ }^{a}$

Alkalinization after 7 days of:

\begin{tabular}{|c|c|c|c|c|c|c|c|c|c|c|c|c|c|c|c|c|c|c|c|c|c|c|c|}
\hline 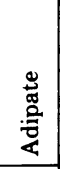 & 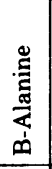 & 营 & 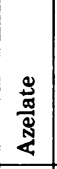 & $\begin{array}{l}\stackrel{\Xi}{0} \\
\stackrel{0}{0} \\
\frac{0}{0}\end{array}$ & 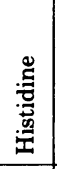 & 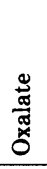 & 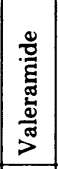 & 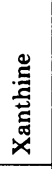 & 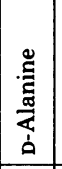 & 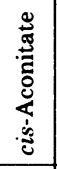 & 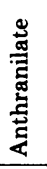 & 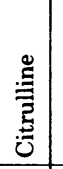 & 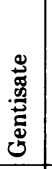 & 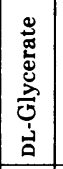 & 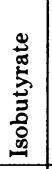 & 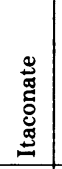 & 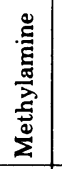 & 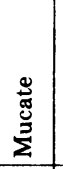 & 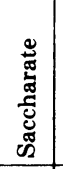 & 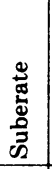 & 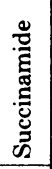 & 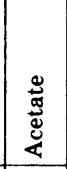 & 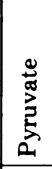 \\
\hline 67 & 67 & 83 & 44 & 39 & 78 & 33 & 78 & 28 & 72 & 33 & 56 & 83 & 0 & 83 & 72 & 79 & 83 & 94 & 89 & 61 & 72 & NT & NT \\
\hline 82 & 49 & 0 & 67 & 91 & 94 & 0 & 18 & 0 & 70 & 91 & 3 & 6 & 91 & 79 & 67 & 76 & 0 & 91 & 88 & 88 & 85 & NT & NT \\
\hline 97 & 80 & 3 & 70 & 80 & 93 & 97 & 80 & 100 & 57 & 100 & 83 & 80 & 7 & 73 & 20 & 97 & 0 & 93 & 90 & 77 & 93 & $\mathbf{N T}$ & NT \\
\hline 61 & 52 & 0 & 52 & 0 & 83 & 0 & 78 & 0 & 65 & 96 & 65 & 61 & 65 & 91 & 78 & 91 & 0 & 91 & 96 & 83 & 87 & NT & NT \\
\hline 92 & 88 & 12 & 16 & 4 & 0 & 0 & 84 & 0 & 92 & 84 & 36 & 88 & 0 & 84 & 80 & 100 & 0 & 96 & 84 & 4 & 84 & NT & NT \\
\hline 54 & 0 & 0 & 15 & 0 & 69 & 0 & 38 & 0 & 77 & 92 & 23 & 8 & 46 & 69 & 8 & 31 & 0 & 100 & 92 & 31 & 77 & NT & NT \\
\hline 73 & 0 & 0 & 55 & 36 & 0 & 0 & 45 & 36 & 0 & 73 & 0 & 0 & 0 & 64 & 73 & 73 & 0 & 0 & 9 & 82 & 27 & NT & NT \\
\hline 0 & 18 & 5 & 5 & 64 & 59 & 5 & 55 & 0 & 59 & 96 & 96 & 0 & 0 & 64 & 55 & & 0 & 5 & 0 & 0 & 82 & NT & NT \\
\hline 7 & 73 & 0 & 0 & 0 & 0 & 0 & 73 & 0 & 67 & 100 & 87 & 0 & 0 & 0 & 0 & 0 & 0 & 0 & 0 & 0 & 0 & NT & NT \\
\hline 0 & 83 & 78 & 0 & 44 & 56 & 0 & 6 & 0 & 61 & 72 & 0 & 72 & 6 & 72 & 11 & 79 & 0 & 11 & & 6 & 0 & NT & NT \\
\hline 17 & 100 & 67 & 17 & 0 & 50 & 0 & 0 & 0 & 100 & 50 & 0 & 100 & 0 & 33 & 67 & 0 & 0 & & 0 & 17 & 17 & NT & NT \\
\hline 0 & 0 & 0 & 17 & 67 & 17 & 17 & 17 & 50 & 17 & 100 & 0 & 17 & 0 & 67 & 17 & 67 & 0 & 83 & 83 & 50 & 17 & NT & NT \\
\hline 7 & 21 & 79 & 7 & 0 & 93 & 7 & 14 & 0 & 57 & 0 & 7 & 64 & 21 & 43 & 50 & 71 & 50 & 79 & 100 & 79 & 93 & NT & NT \\
\hline 0 & 0 & 67 & 0 & 0 & 33 & 0 & 0 & 0 & 67 & 0 & 33 & 100 & 67 & 100 & 66 & 100 & 100 & 67 & 67 & 33 & 0 & NT & NT \\
\hline 0 & 0 & 75 & 0 & 0 & 100 & 0 & 100 & 0 & 75 & 25 & 0 & 100 & 0 & 50 & 75 & 50 & 50 & & 0 & 0 & 75 & NT & NT \\
\hline 70 & 60 & 0 & 10 & 20 & 90 & 0 & 10 & 0 & 20 & 60 & 0 & 50 & 0 & 60 & 0 & 11 & 0 & 10 & 30 & 40 & 50 & NT & NT \\
\hline 10 & 5 & 86 & 14 & 0 & 81 & 29 & 71 & 0 & 57 & 5 & 5 & 91 & 0 & 29 & 0 & 19 & 10 & 43 & 57 & 0 & 33 & NT & NT \\
\hline 100 & 27 & 55 & 100 & 0 & 9 & 0 & 9 & 0 & 100 & 55 & 55 & 9 & 0 & 0 & 27 & 9 & 0 & 0 & 0 & 82 & 9 & NT & NT \\
\hline 14 & 0 & 71 & 14 & 0 & 71 & 0 & 0 & 0 & 71 & 100 & 0 & 0 & 0 & 0 & 29 & 0 & 0 & 0 & 0 & 0 & 0 & NT & NT \\
\hline 10 & 0 & 10 & 20 & 10 & 10 & 10 & 0 & 0 & 50 & 40 & 0 & 20 & 0 & 20 & 0 & 30 & 0 & 0 & 20 & 20 & 30 & NT & NT \\
\hline 0 & 0 & 0 & 0 & 0 & 10 & 10 & 10 & 0 & 0 & 0 & 0 & 20 & 0 & 10 & 0 & 0 & 0 & 10 & 0 & 0 & 0 & NT & NT \\
\hline 0 & 2 & 2 & 0 & 0 & 0 & 0 & 2 & 0 & 45 & 4 & 0 & 0 & 0 & 0 & 11 & 0 & 0 & 0 & 0 & 2 & 0 & 88 & 79 \\
\hline 0 & 0 & 0 & 0 & 0 & 0 & 0 & 0 & 0 & 0 & 0 & 0 & 0 & 0 & 0 & 0 & 0 & 0 & 0 & 0 & 0 & 0 & 0 & 0 \\
\hline 0 & 0 & 0 & 0 & 0 & 0 & 0 & 0 & 0 & 0 & 0 & 0 & 0 & 0 & 0 & 0 & 0 & 0 & 0 & 0 & 0 & 0 & 77 & 73 \\
\hline 0 & 0 & 0 & 0 & 0 & 0 & 0 & 0 & 0 & 20 & 0 & 0 & 0 & 0 & 0 & 10 & 0 & 0 & 0 & 0 & 0 & 0 & 20 & 0 \\
\hline 0 & 0 & 0 & 0 & 0 & 0 & 0 & 0 & 0 & 0 & 0 & 0 & 0 & 0 & 0 & 0 & 0 & 0 & 0 & 0 & 0 & 0 & 0 & 53 \\
\hline 5 & 0 & 5 & 0 & 0 & 0 & 0 & 5 & 0 & 14 & 59 & 0 & 0 & 0 & 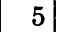 & 0 & 64 & 0 & 0 & 5 & 0 & 5 & NT & NT \\
\hline 0 & 0 & 0 & 0 & 0 & 0 & 0 & 0 & 0 & 0 & 0 & 0 & 0 & 0 & 0 & 0 & 0 & 0 & 0 & 0 & 0 & 0 & 6 & 0 \\
\hline 0 & 0 & 6 & 0 & 0 & 0 & 0 & 0 & 0 & 69 & 69 & 0 & 0 & 13 & 0 & 38 & 94 & 0 & 0 & 6 & 0 & 0 & NT & NT \\
\hline 0 & 0 & 17 & 0 & 0 & 0 & 0 & 17 & 0 & 100 & 83 & 0 & 0 & 0 & 0 & 33 & 67 & 0 & 0 & 0 & 0 & 17 & NT & NT \\
\hline
\end{tabular}

${ }^{a}$ The numbers of strains in the clusters were as follows: cluster I, 18; cluster II, 33; cluster III, 30; cluster IV, 23; cluster V, 25; cluster VI, 13; cluster VII, 11; cluster VIII, 22; cluster IX, 15; cluster X, 18; cluster XI, 6; cluster XII, 6; cluster XIII, 14; cluster XIV, 3; cluster XV, 4; cluster XVI, 9; cluster XVII, 21; cluster XVIII, 11; cluster XIX, 7; cluster XX, 10; cluster XXI, 10; cluster XXII, 47; cluster XXIII, 20; cluster XXIV, 21; cluster XXV, 10; cluster XXVI, 15; cluster XXVII, 22; cluster XXVIII, 18; cluster XXIX, 16; cluster XXX, 6 .

${ }^{b}$ NT, Not tested.

45\% similar were designated taxonomic groupings. The feature frequencies of all characteristics were determined with the feature analysis program FREAK (26), and unique features were determined by using the Krichevsky-Johnson enrichment coefficient.

\section{RESULTS}

A total of 217 organic substrates were examined for substrate utilization, as indicated by alkalinization of the medium by the nonfermenting bacteria chosen for study. Of these substrates, only 58 appeared to have any discriminatory potential. The data concerning the activity on these substrates were incorporated into the numerical analysis study.

A cluster analysis of 165 morphological and biochemical chracteristics of the 541 microorganisms studied revealed 30 distinct clusters at the $45 \%$ level of similarity (Jaccard coefficient). Apparent discrepancies between the number of organisms tested and the number analyzed in the similarity matrix were due to the data processing and analysis programs used. After the data were edited by these programs, 495 strains remained for analysis. The diagrammatic representation of the similarity matrix in Fig. 1 shows the 30 clusters and the intra- and intergroup degrees of similarity. The contents of each clus- 
ter are shown in Table 2. The characteristics most useful for differentiating most of these strains were determined (Table 3). For practical purposes, we assumed that the cluster containing the largest number of organisms of a specific species actually represented that species. Other organisms in this cluster could be present for a variety of reasons. Since many more substrates were tested compared with the number of routine diagnostic tests, this was expected because we were looking at a greater number of characteristics.

Generally, most of the clusters confirmed groupings that had been been determined by conventional methods. However, some clusters varied widely in their characteristics and contained representatives of a number of genera and species. Because of the variability in these clusters, the characteristics tested did not separate all clusters since these clusters were based on mostly negative data. Tables 4 through 6 show a number of characteristics that may be useful for differentiating biochemically similar species.

Recently, Alcaligenes faecalis I and A. faecalis II (clusters IV and IX, respectively) were shown to be separable on the basis of carbon substrate alkalinization patterns (19). Our study confirmed these findings and added four additional substrates (histidine, itaconate, mucate, and suberate) that separated these apparent biotypes. In the previous study, Alcaligenes denitrificans was separable from $A$. faecalis I only on the basis of the nitrate reaction. In this study, we found no additional substrates that aided in the separation of these two species. Interestingly, in gas-liquid chromatographic studies (14), $A$. faecalis and $A$. denitrificans were shown to have similar chromatographic patterns. Alcali. genes odorans (cluster VIII) was distinguished easily from $A$. faecalis II by its ability to reduce nitrite to gas and by its alkalinization of succinamide.

Pseudomonas testosteroni (cluster VIII) and Pseudomonas acidovorans (cluster II) were separated by a number of organic substrates. $P$. acidovorans gave positive (alkaline) reactions wtih each of the following substrates: citrate, gentisate, histidine, mucate, and saccharate.

Of the 17 strains of Pseudomonas alcaligenes used in this study, only 4 formed a distinct cluster (cluster XII). The cluster designated Pseudomonas pseudoalcaligenes (cluster X) contained only 11 of the 17 strains used. Obviously, these two groups were not well defined by the characteristics used. The two clusters were distinguishable by the following reactions: $P$. alcaligenes did not grow on salmonella-shigella agar and did not alkalinize arginine, whereas $P$. pseudoalcaligenes did grow on salmonella-shigella agar and did alkalinize arginine. Conversely, $P$. alcaligenes did alkalinize mucate and saccharate, whereas $P$. pseudoalcaligenes did not.

Pseudomonas putrefaciens (cluster I) was distinguishable easily from other pseudomonads studied, but the two clinical biotypes studied (20) were indistinguishable by the methods used.

TABLE 4. Biochemical and substrate alkalinization reactions that are potentially differential for bacteria that are occasionally difficult to separate

\begin{tabular}{|c|c|c|c|c|c|c|c|c|c|c|c|c|c|c|c|c|c|c|}
\hline \multirow[b]{2}{*}{ Taxon } & \multirow[b]{2}{*}{ Cluster(s) } & \multirow[b]{2}{*}{$\begin{array}{c}\text { Growth on } \\
\text { salmonella- } \\
\text { shigella } \\
\text { agar }\end{array}$} & \multirow[b]{2}{*}{$\begin{array}{c}\text { Nitrate } \\
\text { to } \\
\text { nitrite }\end{array}$} & \multicolumn{15}{|c|}{ Alkalinization after 7 days of: } \\
\hline & & & & 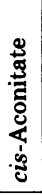 & 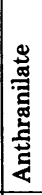 & .气 & 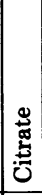 & 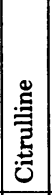 & 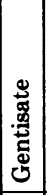 & 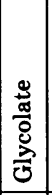 & 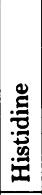 & 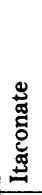 & 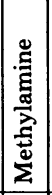 & 总 & 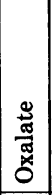 & 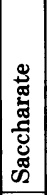 & 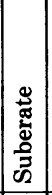 & 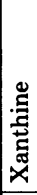 \\
\hline A. denitrificans & VI & $+^{a}$ & + & + & d & - & + & d & d & - & $\mathrm{d}$ & $d$ & - & + & - & + & d & - \\
\hline A. faecalis I & IV & + & d & + & d & - & + & - & d & - & + & + & - & + & - & + & + & - \\
\hline A. faecalis II & IX & + & - & + & + & - & + & - & - & - & - & - & - & - & - & - & - & - \\
\hline P. testosteroni & VII & d & + & d & - & - & - & - & - & d & - & d & - & - & - & - & + & d \\
\hline P. acidovorans & II & d & + & + & - & - & + & - & + & + & + & d & - & + & - & + & + & + \\
\hline$P$. alcaligenes & XII & - & d & + & - & - & - & - & - & d & - & d & - & + & d & + & d & d \\
\hline P. pseudoalcaligenes & $\mathbf{X}$ & + & + & d & - & + & d & d & - & d & d & $\mathrm{d}$ & - & - & - & - & - & - \\
\hline P. putrefaciens & & + & + & d & - & + & - & + & - & d & d & $d$ & + & + & d & + & d & d \\
\hline$P$. diminuta & $\underset{X V}{\text { XIII, XIV, }}$ & - & - & - & d & d & - & + & d & - & + & $d$ & d & d & - & d & d & - \\
\hline B. bronchiseptica & $\mathrm{V}$ & + & + & + & - & - & + & + & - & - & - & + & - & + & - & + & - & - \\
\hline CDC group IVc-2 & III & - & - & + & + & - & + & d & - & + & + & + & - & + & + & + & + & + \\
\hline
\end{tabular}

${ }^{a}+$, More than $80 \%$ of the strains were positive; - , more than $80 \%$ of the strains were negative; $d$, between 20 and $80 \%$ of the strains were positive. 
TABLE 5. Biochemical and substrate alkalinization reactions that are potentially differential for the Moraxella group

\begin{tabular}{|c|c|c|c|c|c|c|c|}
\hline \multirow{2}{*}{ Taxon } & \multirow{2}{*}{ Cluster(s) } & \multirow{2}{*}{$\begin{array}{l}\text { Nitrate } \\
\text { to ni- } \\
\text { trite }\end{array}$} & \multirow{2}{*}{$\begin{array}{l}\text { Phenylala- } \\
\text { nine deam- } \\
\text { inase }\end{array}$} & \multirow{2}{*}{ Urease } & \multicolumn{3}{|c|}{ Alkalinization after 7 days of: } \\
\hline & & & & & Acetate & Itaconate & Pyruvate \\
\hline M. osloensis (CDC group M-5) ${ }^{a}$ & XXII & $-{ }^{b}$ & $\mathrm{~d}$ & - & + & - & + \\
\hline CDC group M-6 & XXIV & + & - & - & + & - & $\mathbf{d}$ \\
\hline M. nonliquefaciens & XXIII & + & - & - & - & - & - \\
\hline M. atlantae & XXVI & - & - & - & - & - & $\mathbf{d}$ \\
\hline M. phenylpyruvica & XXVIII & + & + & + & - & - & - \\
\hline M. urethralis & XXIX, XXX & - & + & - & $\mathbf{N T}^{c}$ & + & NT \\
\hline
\end{tabular}

${ }^{a}$ Numerical taxonomy placed CDC group M-5 and M. osloensis in one cluster.

${ }^{b}+$, More than $80 \%$ of the strains were positive; - , more than $80 \%$ of the strains were negative; $d$, between 20 and $80 \%$ of the strains were positive.

${ }^{c}$ NT, Not tested.

TABLE 6. Biochemical and substrate alkalinization reactions that are potentially differential for the Alcaligenes group and similar organisms

\begin{tabular}{|c|c|c|c|c|c|c|c|c|c|c|}
\hline \multirow[b]{2}{*}{ Taxon } & \multirow[b]{2}{*}{ Cluster(s) } & \multirow[b]{2}{*}{$\begin{array}{l}\text { Nitrite } \\
\text { to gas }\end{array}$} & \multirow{2}{*}{$\begin{array}{l}\text { Growth on } \\
\text { salmonella- } \\
\text { shigella } \\
\text { agar }\end{array}$} & \multicolumn{7}{|c|}{ Alkalinization after 7 days of: } \\
\hline & & & & $\begin{array}{l}\text { cis- } \\
\text { Aconi- } \\
\text { tate }\end{array}$ & $\begin{array}{l}\text { An- } \\
\text { thrani- } \\
\text { late }\end{array}$ & $\begin{array}{l}\text { Argi- } \\
\text { nine }\end{array}$ & $\begin{array}{l}\text { Citrul- } \\
\text { line }\end{array}$ & Mucate & $\begin{array}{c}\text { Sac- } \\
\text { charate }\end{array}$ & $\begin{array}{l}\text { Succi- } \\
\text { namide }\end{array}$ \\
\hline P. denitrificans & XI, XX & $+^{a}$ & - & d & - & - & d & - & - & d \\
\hline CDC group IVe & XXVII & + & - & d & - & - & - & - & - & - \\
\hline A. denitrificans & VI & + & + & + & d & - & d & + & + & d \\
\hline A. faecalis I & IV & - & + & + & d & - & d & + & + & + \\
\hline A. faecalis II & IX & - & + & + & + & - & - & - & - & - \\
\hline A. odorans & VIII & + & + & + & + & - & - & - & - & + \\
\hline F. odoratum & XVII & + & - & - & - & + & + & $\mathbf{d}$ & d & d \\
\hline B. bronchiseptica & V & - & + & + & - & - & + & + & + & + \\
\hline
\end{tabular}

${ }^{a}+$, More than $80 \%$ of the strains were positive; - , more than $80 \%$ of the strains were negative; d, between 20 and $80 \%$ of the strains were positive.

A total of 21 strains of Pseudomonas diminuta were used in this study. Three clusters were formed by the 21 strains (clusters XIII, $\mathrm{XIV}$, and XV), and these clusters were separable by utilization of arabinose and alkalinization of valeramide, saccharate, and succinamide (Table 3). All three clusters had the following four characteristics in common: (i) inability to grow on salmonella-shigella agar; (ii) a negative nitrate reaction; (iii) ability to alkalinize citrulline; and (iv) ability to alkalinize histidine.

Pseudomonas denitrificans also did not cluster as one group; rather, clusters XI and XX both contained stains of $P$. denitrificans. These two clusters were separated by the nitrate reaction, by the ability to alkalinize B-alanine, and by the ability to alkalinize citrulline (Table 3 ). Both clusters reduced nitrate to gas. Other reactions of these two clusters varied. When compared with CDC group IVe (cluster XXVII), the $P$. denitrificans group differed only in the strong urease activity exhibited by CDC group IVe.

The Moraxella group was difficult to separate into distinct species (Table 5). Moraxella atlantae (cluster XXVI) and Moraxella phenyl- pyruvica (cluster XXVIII) are separable by conventional methods, and substrate alkalinization studies did not provide any additional distinguishing characteristics. Moraxella urethralis (cluster XXX) could alkalinize itaconate, whereas all of the other Moraxella species studied could not.

Bordetella bronchiseptica (cluster V) and CDC group IVc-2 (cluster III) are often difficult to separate. Conventional methods differentiate these two groups by the ability of $B$. bronchisep tica to grow on salmonella-shigella agar and by its strong urease activity. Also, B. bronchiseptica reduces nitrate to nitrite, whereas CDC group IVc-2 either does not give a reaction or gives a delayed reaction. We studied five additional substrates that should aid in the differentiation of these two groups (Table 4). CDC group IVc-2 caused alkalinization of glycolate, histidine, oxalate, and xanthine, whereas $B$. bronchiseptica did not alkalinize these substrates. $B$. bronchiseptica alkalinized isobutyrate, whereas CDC group IVc-2 did not (Table $3)$.

Flavobacterium odoratum (cluster XXVII) 
may be difficult to distinguish from similarly pigmented Pseudomonas and Pseudomonaslike bacteria. For this reason, $F$. odoratum is included in Table 6. Pigmentation and the fruity odor characteristically produced by $F$. odoratum are useful differentiating characteristics. Additional differentiating biochemical characteristics included the complete reduction of nitrite to gas and the alkalinization of arginine and citrulline.

Two possible biotypes of Acinetobacter calcoaceticus subsp. lwoffi were apparent (clusters XVIII and XIX). However, these two biotypes do not correspond to the biotypes proposed by basing the separation on partial or beta-like hemolysis (25). These clusters (biotypes) appeared to be distinguishable by alkalinization of adipate, azelate, and suberate (Table 3).

Included in this study were the following two groups of weak oxidizers: Pseudomonas vesicularis and CDC group WO-1. These groups were included since at times they give equivocal reactions in oxidative-fermentative carbohydrate medium. These two groups did form distinct clusters (clusters XXI and XVI, respectively) and were distinguishable from the other clusters.

\section{DISCUSSION}

Clinical and public health bacteriologists often find that differentiation of the nonsaccharolytic bacteria presents a problem because there are few nutritional characteristics to observe. Moreover, it is difficult to determine whether there is more than one group of bacteria within a species when identification is based on only a few characteristics.

There are a number of advantages to using carbon substrate alkalinization tests for studies involving the identification and classification of bacteria. This procedure is standardized by using a defined basal medium (Simmons agar base) that is simple to prepare and can be stored for long periods of time with no apparent loss of sensitivity. Moreover, alkalinization of this basal medium results in a pronounced blue color that is simple to interpret.

The 26 species and groups selected for this study represent predominantly nonsaccharolytic or weakly saccharolytic nonfermentative bacteria. Evaluation of these genera with routine diagnostic tests and carbon substrate alkalinization tests revealed a high degree of variability among and within groups.

When numerical taxonomic methods were applied to the 165 characteristics examined for the 541 strains in the study group, 30 clusters were delineated at the $45 \%$ level of similapity (Jaccard coefficient). According to cluster analysis, the conventional biochemical methods resulted in good groups; i.e., the cluster analysis generally supported the conventional methods. However, although cluster analysis supported the conventional groupings, it was often difficult to find one or two features that distinctly separated the clusters.

Because of the physiological variability of the nonsaccharolytic bacteria, a weighted identification key would be difficult, if not impossible, to construct. This study has proved that a significant number of additional characteristics would be helpful for differentiating the nonsaccharolytic bacteria.

Now that a greater number of characteristics are available to aid in the identification of these organisms, additional research involving deoxyribonucleic acid-deoxyribonucleic acid homologies should be conducted to determine groups based on genotypic similarities. The variability within many of the species studied suggests that a number of biotypes or additional species may exist. Once it has been determined which groupings represent true species, a weighted identification key could be constructed that would be practical in clinical bacteriology laboratories.

\section{LITERATURE CITED}

1. Baumann, P. 1968. Isolation of Acinetobacter from soil and water. J. Bacteriol. 96:39-42.

2. Bottone, E., and J. Allerhand. 1968. Association of mucoid encapsulated Moraxella duplex var. nonliquefaciens with chronic bronchitis. Appl. Microbiol. 16: 315-319.

3. Carson, L. A., M. S. Favero, W. W. Bond, and N. J. Peterson. 1973. Morphological, biochemical, and growth characteristics of Pseudomonas cepacia from distilled water. Appl. Microbiol. 25:476-483.

4. Dupont, H. L., and W. W. Spink. 1969. Infections due to gram-negative organisms: an analysis of 860 patients with bacteremia at the University of Minnesota Medical Center, 1958-1966. Medicine (Baltimore) 48:307-332.

5. Ewing, W. A., and B. R. Davis. 1970. Media and tests for differentiation of Enterobacteriaceae. Center for Disease Control, Atlanta, Ga.

6. Gardner, P., W. B. Griffin, M. N. Swartz, and L. J. Kunz. 1970. Nonfermentative gram-negative bacilli of nosocomial interest. Am. J. Med. 48:735-749.

7. Gilardi, G. L. 1971. Characterization of nonfermentative nonfastidious gram-negative bacteria encountered in medical bacteriology. J. Appl. Bacteriol. 34:623-644.

8. Gilardi, G. L. 1971. Characterization of Pseudomonas species isolated from clinical specimens. Appl. Microbiol. 21:414-419.

9. Gilardi, G. L. 1973. Nonfermentative gram-negative bacteria encountered in clinical specimens. Antonie van Leeuwenhoek J. Microbiol. Serol. 39:229-242.

10. Hardy, P. C., G. M. Ederer, and J. M. Matsen. 1970. Contamination of commercially packaged urinary catheter kits with the pseudomonad EO-1. N. Engl. J. Med. 282:33-35. 
11. Juffs, J. S. 1973. Identification of Pseudomonas spp. isolated from milk produced in southeastern Queensland. J. Appl. Bacteriol. 36:585-598.

12. Koser, S. A. 1923. Utilization of the salts of organic acids by the colon-aerogenes group. J. Bacteriol. 8:493-520.

13. Krichevsky, M. 1977. Coding and management of microbiological data. Dev. Ind. Microbiol. 18:309-318.

14. Moss, C. W., and S. B. Dees. 1975 . Cellular fatty acids of Alcaligenes and Pseudomonas species isolated from clinical specimens. J. Clin. Microbiol. 1:414-419.

15. Oberhofer, T. R., and J. W. Rowen. 1974. Acetamide agar for differentiation of nonfermentative bacteria. Appl. Microbiol. 28:720-721.

16. Oberhofer, T. R., J. W. Rowen, and G. F. Cunningham. 1977. Characterization and identification of gram-negative, nonfermentative bacteria. J. Clin. Microbiol. 5:208-220.

17. Otton, L. A., and M. J. Pickett. 1976. Rapid method for identification of gram-negative, nonfermentative bacilli. J. Clin. Microbiol. 3:566-575.

18. Pinter, M., and M. Kantor. 1974. Comparison of Alcaligenes faecalis and Alcaligenes odorans var. viridans by carbon source utilization tests. Acta Microbiol. Acad. Sci. Hung. 21:293-295.

19. Rarick, H. R., P. S. Riley, and R. Martin. 1978. Carbon substrate studies of some cultures of Alcaligenes deni. trificans, Alcaligenes faecalis, and Alcaligenes odorans isolated from clinical specimens. J. Clin. Microbiol. 8:313-319.

20. Riley, P. S., H. W. Tatum, and R. E. Weaver. 1972. Pseudomonas putrefaciens isolated from clinical spec- imens. Appl. Microbiol. 24:798-800.

21. Rogosa, M., M. E. Krichevsky, and R. R. Colwell. 1971. Method for coding data on microbial strains for computer manipulation. Int. J. Syst. Bacteriol. 21(Suppl.):1A-184A.

22. Simmons, J.S. 1926. A culture medium for differentiating organisms of typhoid-colon aerogenes groups and for isolation of certain fungi. J. Infect. Dis. 39:209-214.

23. Snell, J. J., and S. P. Lapage. 1973. Carbon source utilization tests as an aid to the classification of nonfermenting gram-negative bacteria. J. Gen. Microbiol. 74: 9-20.

24. Stanier, R. Y., N. J. Paleroni, and M. Doudoroff. 1966. The aerobic pseudomonads: a taxonomic study. J. Gen. Microbiol. 43: 159-271.

25. Tatum, H. W., W. H. Ewing, and R. E. Weaver. 1974. Miscellaneous gram-negative bacteria, p. 270-294. In E. H. Lennette, E. H. Spaulding, and S. P. Truant (ed.), Manual of clinical microbiology, 2nd ed. American Society for Microbiology, Washington, D.C.

26. Walczak, C. A., R. Johnson, and M. I. Krichevsky. 1978. A flexible approach in phenotypic feature frequency calculation. Program 156. American Society for Microbiology, Washington, D.C.

27. Walczak, C. A., and M. I. Krichevsky. 1977. Interactive computer editing of phenetic data for numerical taxonomy. Program 138. American Society for Microbiology, Washington D.C.

28. Weiss, B. F., H. N. Munro, and R. J. Wurtman. 1971. Pseudomonas aeruginosa growth in distilled water from hospitals. Science 173:836-837. 